\title{
Influência das cultivares de videiras (Vitis vinifera L.) e do sistema de condução sobre a acarofauna
}

\author{
Rita T. L. da Silva ${ }^{1,2}{ }^{\oplus}$, Amália L. W. Berté ${ }^{2 \oplus}$, Gabriel L. Bizarro ${ }^{2 \oplus}$, Angelica Sulzbach ${ }^{2 \oplus}$, Vinicius \\ L. da Silva ${ }^{2 \oplus}$, Noeli J. Ferla ${ }^{1,2,3 \oplus}$, Guilherme L. da Silva ${ }^{2 \oplus}$, Liana Johann ${ }^{2:=}$
}

${ }^{1}$ Programa de Pós-Graduação em Biotecnologia, Universidade do Vale do Taquari - UNIVATES, Lajeado, Rio Grande do Sul, Brasil. ${ }^{2}$ Laboratório de Acarologia, Universidade do Vale do Taquari - UNIVATES, Lajeado, Rio Grande Sul, Brasil. ${ }^{3}$ CNPq Pesquisador. 拝=Corresponding author: liana@univates.br

Edited by: Peterson Rodrigo Demite

Received: March 23, 2021. Accepted: July 02, 2021. Published: August 19, 2021.

Influence of grapevine cultivars (Vitis vinifera L.) and training systems on mite fauna

Abstract. The South of Brazil is the largest producer of grapes, however this production is affected by climate and herbivores such as phytophagous mites. The aim of this work was to associate the mite fauna with the grape cultivars Chardonnay, BRS Lorena and hybrid cultivars rootstock Paulsen 1103, in trellising and espalier systems, in the municipalities of Bento Gonçalves and Garibaldi, Rio Grande do Sul State, Brazil. The cultivars were: Chardonnay trellis $(\mathrm{Cl})$ Chardonnay espalier (Ce), BRS Lorena trellis (LI), BRS Lorena espalier (Le) and the hybrid cultivars Paulsen rootstock $1103(\mathrm{~Pa})$. Monthly samplings were carried out from November 2016 to April 2017. Twenty plants per area were randomly sampled, from each plant three apical leaves were collected, apical, median and basal branches (60 leaves / area). In total, 6,865 mites belonging to seven families, 13 genera and 22 species were collected. The most abundant phytophagous belong to Tarsonemidae and Tenuipalpidae, the predators to Phytoseiidae and the generalists to Tydeidae and Iolinidae. The cultivars Lorena $L /$ was the greatest similarity (82.39\%) among species mites, followed by Paulsen $\mathrm{Pa}(72.54 \%)$, Lorena Le (70.67\%), Chardonnay $\mathrm{Cl}(31.79 \%)$ and Chardonnay Ce (31.48\%). The highest dissimilarity among the cultivars occurred between Chardonnay $\mathrm{Ce}$ and Lorena Le (66.49\%), followed by Chardonnay Ce, Chardonnay $\mathrm{Cl}(66.44 \%)$, Chardonnay $\mathrm{Ce}$ and Lorena $\mathrm{LI}(61.49 \%)$. Thus, there is homogeneity between the sampled cultivars and the conduction systems and the apical third of the leaves and the sampling period are important in determining the composition of the species.

Keywords: Broad mite, Phytophagous mites, Systems.

A videira (Vitis vinifera L.) é cultivada em diferentes regiões do mundo, tendo sido introduzida no Brasil no século XVI e se expandido para o Sul em 1662. Hoje, o estado do Rio Grande do Sul (RS) é o maior produtor de uvas do país, com uma safra anual de 614,2 toneladas (Ibravin 2019). As intempéries (chuva e o granizo) podem colocar em risco o rendimento da cultura, assim como o aparecimento de herbívoros e ácaros fitófagos, como os pertencentes às famílias Tarsonemidae, Eriophyidae, Tenuipalpidae e Tetranychidae (Duso \& Lillo 1996)

O tarsonemídeo Polyphagotarsonemus latus (Baker, 1904), conhecido como ácaro branco, é o ácaro fitófago mais abundante e dominante no cultivo de videiras no Rio Grande do Sul, tendo seu desenvolvimento favorecido quando existe baixa luminosidade, temperatura e umidade alta. É encontrado com maior frequência nas folhas novas e brotações (Da Silva et al. 2020), do qual alimenta-se, ocasionado a paralisação do crescimento dos ramos (Haji et al. 2001).

Além de ácaros fitófagos e generalistas (Tydeidae e lolinidae), são observados ácaros predadores, como os pertencentes às famílias Phytoseiidae e Stigmaeidae. Entre os predadores associados ao ácaro branco em diferentes culturas distribuídas pelo Brasil, os mais comuns são Phytoseiidae, sendo frequentemente empregados no controle de ácaros que podem alcançar o status de praga (Duso \& Lillo 1996).

No Rio Grande do Sul, as videiras são plantadas utilizando-se dois sistemas de condução da planta: a) espaldeira - crescimento na vertical; b) latada - crescimento na horizontal. Entretanto, as informações são bem restritas sobre os sistemas de condução das videiras, e das cultivares, sobre as espécies de ácaros fitófagos e predadores. Com a hipótese de que o sistema de condução latada seja mais favorável para ao desenvolvimento de $P$. latus, por ter a poda verde dificultada, este trabalho teve por objetivo conhecer a acarofauna em folhas apicais associada às videiras viníferas Chardonnay, BRS Lorena e cultivar híbrida porta-enxerto Paulsen 1103, nos sistemas de condução latada e espaldeira, nos municípios de Bento Gonçalves e Garibaldi, no estado do Rio Grande do Sul, Brasil.

O estudo foi conduzido em vinhedos localizados nos municípios de Bento Gonçalves, com as cultivares viníferas: Chardonnay latada $(\mathrm{Cl})$ $(0,7$ hectare, 8 anos de idade), Chardonnay espaldeira $(\mathrm{Ce})$ (1 hectare, 8 anos de idade) $\left(29^{\circ} 11^{\prime} 43.4^{\prime \prime} \mathrm{S} 51^{\circ} 36^{\prime} 47.2^{\prime \prime} \mathrm{W}\right)$ e BRS Lorena latada (LI) (0,4 hectare, 10 anos de idade) ( $\left.29^{\circ} 11^{\prime} 32.0^{\prime \prime} \mathrm{S} 51^{\circ} 36^{\prime} 39.4^{\prime \prime} \mathrm{W}\right)$; e de Garibaldi: BRS Lorena espaldeira (Le) (0,2 hectare, 10 anos de idade) $\left(29^{\circ} 15^{\prime} 02.5^{\prime \prime} \mathrm{S} 51^{\circ} 38^{\prime} 46.6^{\prime \prime} \mathrm{W}\right)$ e a cultivar híbrida porta-enxerto Paulsen $1103(P a)\left(0,4\right.$ hectare, 1 ano de idade) (29 $\left.11^{\prime} 13.7^{\prime \prime S} 51^{\circ} 38^{\prime} 09.6^{\prime \prime} \mathrm{W}\right)$. Ambos os municípios estão localizados no Vale dos Vinhedos, no estado do Rio Grande do Sul, Brasil e inseridos na Formação da Floresta Estacional Semidecidual com tipo de clima subtropical ( $C f a)$, que se caracteriza como moderado chuvoso (Peel et al. 2007). Durante o período de coleta observou-se temperatura média do ar de $20,80^{\circ} \mathrm{C}$ $\left(18,2^{\circ} \mathrm{C}-22,8^{\circ} \mathrm{C}\right)$, umidade relativa do ar média de $76,37 \%(70,4 \%$ $81,4 \%)$ e precipitação pluviométrica média de $171,33 \mathrm{~mm}(102 \mathrm{~mm}$ $260 \mathrm{~mm})$.

As coletas, quinzenais, foram realizadas no período de novembro de 2016 a abril de 2017, mantendo-se o mesmo tratamento convencional em todas as áreas, sem aplicação de acaricida. De cada área, foram amostradas aleatoriamente 20 plantas em uma fileira, demarcada com cordas de ráfia, sem realização de poda verde. De cada planta foram coletadas três folhas apicais, sendo uma do ramo apical, uma do ramo mediano e outra do ramo basal, totalizando 60 folhas por área.

As folhas das cultivares coletadas foram individualizadas em sacos plásticos previamente identificados com o nome da cultivar e área, acondicionadas em caixa de isopor com Gelox ${ }^{\circledR}$ (para manter a baixa temperatura) e levadas para o laboratório para triagem (contagem e retirada de ácaros) sob microscópio estereoscópico, observando-se 
as duas faces das folhas. Os indivíduos foram montados em lâminas utilizando meio Hoyer (Jeppson et al. 1975), após foram mantidas em estufa a $50-60^{\circ} \mathrm{C}$ por um período de sete dias para a secagem do meio. Posteriormente as lâminas foram lutadas, etiquetadas, acondicionadas em caixas porta lâminas e mantidas em sala com umidade relativa menor que $40 \%$.

A identificação dos espécimes foi realizada com o auxílio de microscópio óptico com contraste de fase e chaves dicotômicas (Chant \& McMurtry 1994; Amrine Jr. et al. 2003; Fan \& Zhang 2005; Chant \& McMurtry 2007; Johann et al. 2013; Skvarla et al. 2014; Da Silva et al. 2016; Johann et al. 2017).

Para a ordenação das diferentes cultivares e suas respectivas localidades com base na abundância da acarofauna associada, foi realizada uma Análise de Escalonamento Multidimensional Não Métrica (NMDS). Esses dados foram utilizados com uma matriz de similaridade Bray-Curtis. A fim de verificar diferenças significativas na composição dos ácaros dos grupos ordenados pela NMDS, foi realizada uma Análise de Similaridade (ANOSIM) com 999 permutações utilizando dados de abundância. Realizou-se, ainda, a Análise de Porcentagem de Similaridade (SIMPER) para identificar as espécies que mais contribuíram para a similaridade e a dissimilaridade entre as diferentes cultivares. As ordenações NMDS e ANOSIM, assim como a SIMPER, foram realizadas no software PRIMER-E (Clarke \& Gorley 2006) versão 5.2.9. (Metodologia utilizada por Da Silva et al. 2020).

Um dendrograma de similaridade entre as comunidades acarinas das diferentes cultivares foi realizado aplicando-se o índice de Jaccard. Tal dendrograma resultou de uma análise de agrupamento utilizando o algoritmo UPGMA (Unweighted Pair-Group Method using Arithmetic Averages) e o índice de Sørensen (Bray-Curtis) (Gotelli \& Ellison 2011) no programa estatístico Paleontological Statistics (Hammer et al. 2001) versão 3.0.

Diferente do esperado, observou-se que a cultivar tem mais influência sobre a abundância de ácaros do que o sistema de condução. A cultivar Lorena Le apresentou maior abundância acarina 2.481 espécimes, seguida de Paulsen $\mathrm{Pa}$ (2.433 espécimes), Chardonnay $\mathrm{Cl}$ (1.103 espécimes), Lorena LI (740 espécimes) e Chardonnay Ce (108 espécimes).

Foram coletados em $V$. vinifera e videira híbrida um total de 6.865 ácaros pertencentes a 22 espécies, distribuídas em 13 gêneros e sete famílias (Fig. 1). Os fitófagos mais abundantes foram Tarsonemidae e Tenuipalpidae. Em Tarsonemidae, P. latus foi a espécie mais abundante (6.547 espécimes), seguido de Tarsonemus sp. (85 espécimes). Já entre os Tenuipalpidae, a espécie Brevipalpus obovatus (Donnadieu, 1875) foi a mais abundante (14 espécimes).

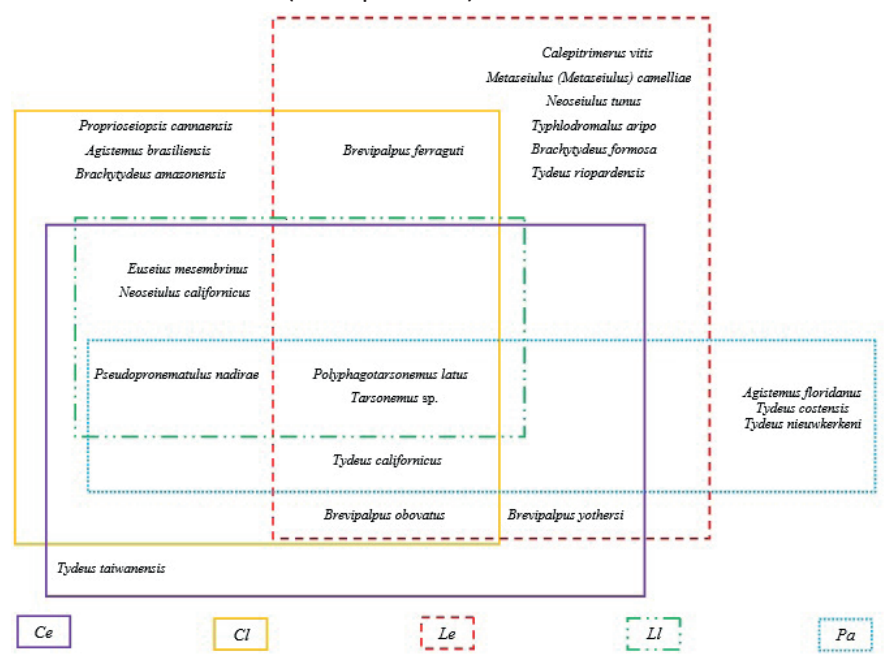

Figura 1. Ácaros associados com as cultivares Chardonnay latada $(\mathrm{Cl})$, Chardonnay espaldeira (Ce), BRS Lorena latada (LI), BRS Lorena espaldeira (Le) e a cultivar híbrida Porta-enxerto Paulsen 1103 (Pa), entre novembro de 2016 e abril de 2017, nos municípios de Bento Gonçalves e Garibaldi, no estado do Rio Grande do Sul, Brasil.

Os ácaros predadores mais abundantes foram representados pelos Phytoseiidae (63 espécimes) e entre os generalistas Iolinidae (56) e
Tydeidae (43). Em Phytoseiidae, as espécies mais abundantes foram Neoseiulus californicus (McGregor, 1954), com 53 espécimes, Euseius mesembrinus (Dean, 1957), com 32 espécimes e Typhlodromalus aripo DeLeon, 1967, com 18 espécimes. Na família lolinidae, a espécie mais abundante foi Pseudopronematulus nadirae Da Silva, Da-Costa \& Ferla, 2017, com 56 espécimes, e em Tydeidae, Tydeus californicus (Banks, 1904), com 16 espécimes.

A comparação das comunidades acarinas encontradas nas cultivares viníferas e híbrida mostraram que sistema de condução, ramo apical e o período das coletas podem ter interferido na maior ou menor similaridade. As espécies acarinas observadas associadas a cultivar Lorena Le (1) no mês de novembro, separaram-se prontamente das demais espécies, formando um bloco menor, apresentando somente $15 \%$ de similaridade entre esses organismos quando comparadas com as demais cultivares (Fig. 2).

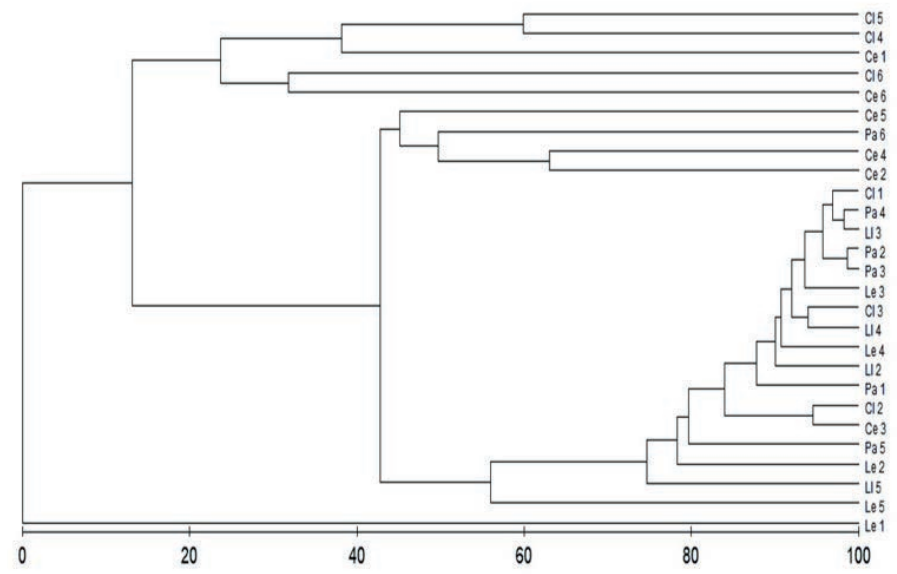

Similaridade

Figura 2. Dendrograma de similaridade para as espécies acarinas coletadas em ramos apicais das cultivares viníferas Chardonnay latada $(C l)$, Chardonnay espaldeira $(\mathrm{Ce})$ e BRS Lorena latada ( $L I)$, BRS Lorena espaldeira (Le) e a cultivar híbrida Porta-enxerto Paulsen $1103(\mathrm{~Pa})$, onde os números 1 a 6 representam os meses de novembro a abril, localizadas nos municípios de Bento Gonçalves e Garibaldi, no estado do Rio Grande do Sul, Brasil.

Ao considerar todas as espécies acarinas encontradas nas cultivares, a Análise de Similaridade agrupou as cultivares Chardonnay $\mathrm{Ce}(1,2,3$, 4, 5 e 6), Chardonnay $\mathrm{Cl}(1,2,3,4,5$ e 6), Paulsen $\mathrm{Pa}(1,2,3,4,5$ e 6), Lorena $L I(1,2,3,4,5$ e 6$)$ e Lorena Le (2, 3, 4, 5 e 6), que apresentaram maior similaridade entre si $(85 \%)$, demonstrando semelhança na composição das espécies, ou seja, as cultivares compartilharam as mesmas espécies.

A acarofauna presente nas cultivares é constituída, em sua grande maioria, por ácaros fitófagos, especialmente por P. latus. Normalmente, esses ácaros apresentam alta taxa de crescimento populacional, podendo alcançar o status de praga e ocasionar prejuízos econômicos na cultura (Moraes \& Flechtmann 2008).

A cultivar Lorena $L /$ apresentou maior similaridade no que se refere a semelhança da acarofouna (82,39\%), seguida das cultivares Paulsen $\mathrm{Pa}(72,54 \%)$, Lorena Le (70,67\%), Chardonnay Cl (31,79\%) e Chardonnay $\mathrm{Ce}(31,48 \%)$. Sendo N. californicus, E. mesembrinus, P. nadirae e P. latus as espécies acarinas que mais contribuíram para tal similaridade.

Através da SIMPER, verificou-se que o percentual de similaridade entre as cultivares avaliadas foi decrescente da cultivar Lorena $\mathrm{LI}$ para a Paulsen $P a$ e para a Lorena Le. Entre as cultivares Lorena $L$ I e Paulsen $P a$, P. latus $(97,97 \%$ e $90,88 \%$, respectivamente) foi a espécie acarina que mais contribui para essa homogeneidade; $P$. latus $(62,62 \%)$, E. mesembrinus $(14,56 \%), N$. californicus $(11,65 \%)$ e $P$. nadirae $(6,74 \%)$, foram os ácaros que mais contribuíram para a similidaridade em Chardonnay $\mathrm{Cl}$. Já em Chardonnay $\mathrm{Ce}$ a semelhança foi devido à ocorrência de $P$. latus (42,53\%), P. nadirae $(32,94 \%)$ e $N$. californicus $(21,43 \%)$. A mesma análise evidenciou que a maior média de dissimilaridade entre as cultivares ocorreu entre Chardonnay $\mathrm{Ce}$ e Lorena Le $(66,49)$, seguida de Chardonnay Ce e Chardonnay Cl (66,44\%). Polyphagotarsonemus latus $(26,38 \%)$ e P. nadirae 
$(21,08 \%)$ contribuíram para maior dissimilaridade entre as cultivares Chardonnay $\mathrm{Ce}$ e Lorena Le. Entre Chardonnay $\mathrm{Ce}$ e Chardonnay $\mathrm{Cl}$, as espécies que mais contribuíram para maior dissimilaridade foram $P$. latus $(34,36 \%)$ e $P$. nadirae (13,55\%), enquanto na comparação entre as cultivar Chardonnay Ce e Lorena L/ foram P. latus $(31,50 \%)$ e $P$. nadirae (24,03\%).

As cultivares Chardonnay $\mathrm{Cl}$, Chardonnay $\mathrm{Ce}$, Lorena $\mathrm{LI}$, Lorena Le e Paulsen $P a$, através da análise NMDS foram agrupadas conforme a abundância e riqueza acarina, de modo que cada uma delas representa um local amostrado. O valor de "perturbação" fornecido no escalonamento (valor de Stress $=0,09$ ) indica que a análise corresponde a uma boa ordenação (Fig. 3), resultado foi confirmado pela análise ANOSIM ( $R$ global $=0,061 p=>0,005)$. Verificou-se as áreas amostradas são constituídos na sua maioria pelas mesmas espécies acarinas.

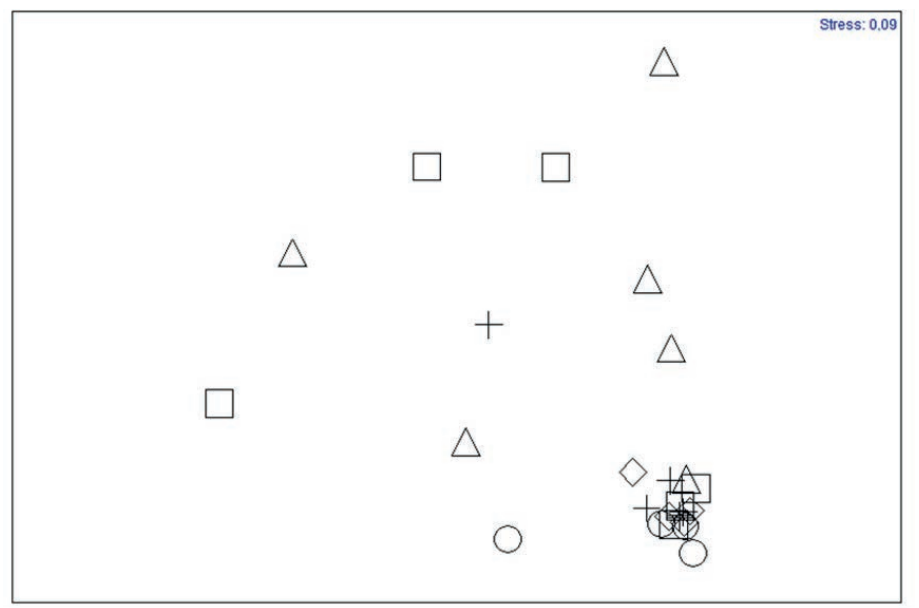

Figura 3. Representação gráfica da ordenação resultante da análise de escalonamento multidimensional não métrico (NMDS), com base na presença das espécies acarinas dos cinco sítios: Chardonnay $\mathrm{Ce}(\Delta)$, Chardonnay $\mathrm{Cl}(\square)$, Lorena Le $(0)$, Lorena $L I(\diamond)$ e Paulsen $P a(+)$, associadas às cultivares viníferas e híbridas localizadas nos municípios de Bento Gonçalves e Garibaldi, no estado do Rio Grande do Sul, Brasil. Stress=0,09.

Polyphagotarsonemus latus foi a espécie acarina mais frequente e abundante em todas as cultivares avaliadas, representando $95 \%$ de todos os ácaros coletados, sendo observada com maior frequência na face abaxial das folhas, com maiores populações entre os meses de janeiro e fevereiro. Esta espécie é observada frequentemente associada a diferentes culturas (Sarmento et al. 2011), entre estas a videira. Alimenta-se das folhas apicais (novas) e brotações (Haji et al. 2001). No Vale do São Francisco, P. latus é uma das principais pragas associadas às videiras, causando perdas significativas devido à atrofia dos ramos (Haji et al. 2001). Em levantamento realizado no município de Bento Gonçalves, P. latus foi observado nas folhas apicais e associado a cultivar Cabernet Sauvignon (Johann et al. 2009). Klock et al. (2011) reportaram $P$. latus associado a cultivar Merlot. Em levantamento realizado nos municípios de Dois Lajeados, Imigrante, Marques de Souza e Putinga, P. latus foi observado com maior frequência e abundância nas folhas apicais, associado a todas as cultivares viníferas avaliadas, sem contudo, ocasionar prejuízo à cultura (Da Silva et al. 2020).

Dentre os ácaros predadores, Phytoseiidae foram os mais abundantes, associados às cultivares Chardonnay $\mathrm{Ce}$ e Chardonnay $\mathrm{Cl}$, destacando-se as espécies $N$. californicus e $E$. mesembrinus. Os fitoseídeos são habitualmente utilizados para controlar ácaros fitófagos e insetos em diferentes culturas (Simoni \& Castagnoli 2010).

Neoseiulus californicus foi observado com maior frequência associado a cultivar Chardonnay $\mathrm{Ce}$ na face abaxial das folhas, em maiores populações no mês de dezembro/16. Este ácaro foi encontrado com frequência e abundância associado às cultivares Alfrocheiro, Cabernet Sauvignon e Pinot Noir nos municípios de Bento Gonçalves, Candiota e Encruzilhada do Sul (Johann et al. 2009). Alimenta-se de tetraniquídeos, outros artrópodes, seiva vegetal e principalmente de pólen, sendo utilizado para controlar ácaros fitófagos (McMurtry et al. 2013).

Euseius mesembrinus foi observado com maior frequência associado a cultivar Chardonnay $\mathrm{Cl}$, na face abaxial das folhas, em maiores populações no mês de abril/17, associado a $P$. latus. Esse fitoseídeo é generalista, alimentando-se de ácaros e de pólen, e apresenta potencial como possível inimigo natural de ácaros fitófagos (McMurtry et al. 2013). Em estudo realizado nos municípios de Dois Lajeados, Imigrante, Marques de Souza e Putinga, E. mesembrinus foi observado com maior frequência nas folhas apicais da cultivar vinífera Rainha Itália (Da Silva et al. 2020).

As análises de similaridade mostraram que a acarofauna das cultivares viníferas e híbrida de cada ambiente avaliado são semelhantes. A comparação das comunidades de ácaros encontradas nessa cultura gerou dois agrupamentos/blocos distintos. O bloco maior é representado pelas cultivares que apresentam maior similaridade, ou seja, que compartilham as mesmas espécies acarinas. Cabe ressaltar que ocorreu este agrupamento devido às espécies $P$. latus, $N$. californicus, $E$. mesembrinus e $P$. nadirae, que foram observadas frequentemente nas cultivares avaliadas. Este evento sugere que essa similaridade tenha ocorrido devido aos ácaros predadores estarem associados à sua fonte de alimento, no caso, os tarsonemídeos, reforçando a relação predador-presa. O bloco menor é representado pela cultivar Lorena Le (1), que apresenta menor similaridade, ou seja, esta cultivar compartilha poucas espécies acarinas com as demais cultivares, entre essas $P$. latus.

Os agrupamentos gerados pelo cluster foram confirmados através da análise de similaridade (ANOSIM) e pela análise de agrupamento (NMDS), ambas análises reforçam a alta similaridade entre os blocos e indicam que os sistemas de condução, terço das folhas (apical) e o período das coletas tiveram influência sobre a composição e a abundância das comunidades acarinas, sendo importantes na determinação da composição das espécies.

Devido à importância da vitivinicultura para a economia do Rio Grande do Sul, principalmente no Vale dos Vinhedos, a realização de estudos oportuniza reconhecer espécies acarinas da família Phytoseiidae promissoras no controle de ácaros fitófagos e, por conseguinte, estabelecer estratégias adequadas de manejo dos vinhedos que permitam a manutenção desses inimigos naturais na cultura da videira, para que possam atuar no controle de espécies acarinas fitófagas.

\section{Agradecimentos}

Os autores agradecem a Universidade do Vale do Taquari - Univates pelo financiamento da pesquisa e concessão das bolsas de iniciação científica e a Vinícola Garibaldi pela oportunidade de realizar as coletas nas suas áreas de produção de uvas viníferas.

\section{Contribuições dos autores}

Conceptualização: LJ, GLS; Coleta e identificação dos ácaros: ALWB, GLB, RTLS; Redação e edição: RTLS, ALWB, NJF; Análises dos dados: GLS, VLS; Correção final do manuscrito: LJ, AS.

\section{Referências}

Amrine Jr, J. W.; Stasny, T. A.; Flechtmann, C. H. (2003) Revised keys to world genera of Eriophyoidea (Acari: Prostigmata). West Bloomfield: Indira Publishing House.

Chant, D. A.; McMurtry, J. A. (1994) A review of the subfamilies Phytoseiinae and Typhlodrominae (Acari: Phytoseiidae). International Journal of Acarology, 20(4): 223-310. doi: 10.1080/01647959408684022

Chant, D. A.; McMurtry, J. A. (2007) Illustrated keys and diagnoses for the genera and subgenera of the Phytoseiidae of the world (Acari: Mesostigmata). West Bloomfield: Indira Publishing House.

Clarke, K.R.; Gorley, R.N. (2006) PRIMER-E v6: user manual/tutorial. Plymouth: PRIMER-E.

Da Silva, G. L.; Metzelthin, M. H.; Da Silva, O. S.; Ferla, N. J. (2016) Catalogue of the mite family Tydeidae (Acari: Prostigmata) with the world key to the species. Zootaxa, 4135(1): 1-68. doi: 10.11646/ 
zootaxa.4135.1.1

Da Silva, R. T. L.; Da Silva, V. L.; Silva, D. E.; Do Nascimento, J. M.; Schüssler, M.; Spies, F. F.; Berté, A. L. W.; Da Silva, G. L.; Johann L.; Ferla, J. J. (2020) Bioecological aspects of mites associated with Vitis vinifera varieties in the state of Rio Grande Do Sul, Brazil. Systematic and Applied Acarology, 25(9): 1618-1642. doi: 10.11158/saa.25.9.8

Duso, C.; Lillo, D. C. M. (1996) Grape. In: Lindquist, E. E., Bruin, J.; Sabelis, M. W. (Eds.), Eriophyid mites: their biology, natural enemies and control. Amsterdam: Elsevier.

Fan, Q. H.; Zhang, Z. Q. (2005) Raphignathoidea (Acari: Prostigmata). Fauna of New Zealand, 52. doi: 10.7931/J2/FNZ.52

Gotelli, N.J.; Ellison, A. M. (2011) Princípios de estatística em ecologia. Artmed: Porto Alegre.

Haji, F. N. P.; Moreira, A. N.; Lopes, P. R. E.; Ferreira, R. E. F. Alencar J.A.; Barbosa, F. R. (2001) Monitoramento e determinação do nível de ação do ácaro-branco na cultura da uva. Embrapa Semiárido, 7p. (EMBRAPA Semiárido, Circular Técnica, 68).

Hammer O.; Harper, D.A.T.; Rian, P.D. (2001) Past: Palaeonthological statistics software package for education and data analysis. Palaeontologia electronica, 4: 1-9.

Ibravin - Instituto Brasileiro do Vinho. Uvas processadas no RS Milhões de $\mathrm{kg}$. https://www.ibravin.org.br/admin/arquivos/ estatisticas/1561748795.pdf. Acesso em: viii.2019.

Jeppson, L. R.; Keifer, H. H.; Baker, E. W. (1975) Mites injurious to economic plants. Berkeley: University of California Press.

Johann, L.; Carvalho, G. S.; Dos Santos R. M.; Ferla, N. J. (2013) A new species of Agistemus (Acari: Stigmaeidae) from yerba mate in the state of Rio Grande do Sul, Brazil. International Journal of Acarology, 39(7): 580-586. doi: 10.1080/01647954.2013.856469

Johann, L.; Da Silva, G. L.; Brentano, A. C.; Carvalho, G. S.; Botton, M.; Ferla, N. J. (2017) Chave ilustrada para identificação da fauna acarina na cultura da videira do estado do Rio Grande do Sul, Brasil. Embrapa Uva e Vinho, 16p. (Embrapa Uva e Vinho, Comunicado Técnico, 197).

Johann, L.; Klock, C. L.; Ferla, N. J.; Botton, M. (2009) Acarofauna (Acari) associada à videira (Vitis vinifera L.) no Estado do Rio Grande do Sul. Biociências, 17(1): 1-19.

Klock, C.L.; Johann, L.; Botton, M.; Ferla, N. J. (2011) Mitefauna (Arachnida: Acari) associated to grapevine, Vitis vinifera L. (Vitaceae), in the municipalities of Bento Gonçalves and Candiota, Rio Grande do Sul, Brazil. Check List, 7(4): 522-536. doi: 10.15560/7.4.522

McMurtry, J. A.; Moraes, G. J.; Sourassou, N. F. (2013) Revision of the lifestyles of phytoseiid mites (Acari: Phytoseiidae) and implications for biological control strategies. Systematic and Applied Acarology, 18(4): 297-320. doi: 10.11158/saa.18.4.1

Moraes, G. J.; Flechtmann, C. H. W. (2008) Manual de acarologia: acarologia básica e ácaros de plantas cultivadas no Brasil. Ribeirão Preto: Holos.

Peel, M. C.; Finlayson, B. L.; McMahon, T. A. (2007) Updated world map of the Köppen-Geiger climate classification. Hydrology and Earth System Sciences, 11: 1633-1644. doi: 10.5194/hess-11-1633-2007

Sarmento, R.A.; Rodrigues, D. M.; Faraji, F.; Erasmo, E. A. L.; Lemos, F.; Teodoro, A.V.; Kikuchi, W. T.; Santos, G. R.; Pallini, A. (2011) Suitability of the predatory mites Iphiseiodes zuluagai and Euseius concordis in controlling Polyphagotarsonemus latus and Tetranychus bastosi on Jatropha curcas plants in Brazil. Experimental and Applied Acarology, 53(3): 203-214. doi: 10.1007/s10493-010-9396-2

Simoni, S.; Castagnoli, M. (2010) IPM strategies through specialist and generalist phytoseiids (Acari, Mesostigmata). In Ciancio, A.; Mukerji, k. G. Integrated Management of Arthropod Pests and Insect Borne Diseases, pp. 311-325. Dordrecht: Springer. doi: 10.1007/978-90-481-8606-8_12

Skvarla, M. J.; Fisher, J. R.; Dowling, A. P. G. (2014) A review of Cunaxidae (Acariformes, Trombidiformes): Histories and diagnoses of subfamilies and genera, keys to world species, and some new locality records. ZooKeys, 418: 1-103. doi: 10.3897/ zookeys.418.7629 